FORMATION Formation emploi

Revue française de sciences sociales

134 | Avril-Juin

Pêle-mêle

\title{
Edito : Aux frontières des catégories
}

Jean-Frédéric Vergnies

\section{(2) OpenEdition}

Journals

Édition électronique

URL : http://journals.openedition.org/formationemploi/4712

DOI : 10.4000/formationemploi.4712

ISSN : 2107-0946

\section{Éditeur}

La Documentation française

Édition imprimée

Date de publication : 21 juin 2016

Pagination : 1-2

ISSN : 0759-6340

\section{Référence électronique}

Jean-Frédéric Vergnies, «Edito : Aux frontières des catégories », Formation emploi [En ligne], 134 | AvrilJuin, mis en ligne le 21 juin 2016, consulté le 30 octobre 2020. URL : http://journals.openedition.org/ formationemploi/4712 ; DOI : https://doi.org/10.4000/formationemploi.4712 


\title{
Aux frontières des catégories
}

\author{
Jean-Frédéric Vergnies \\ Rédacteur en chef
}

Ce numéro de Formation Emploi nous invite à revisiter certaines catégories et leurs frontières afin d'ouvrir des horizons plus larges ${ }^{1}$ pour nous aider à penser et agir.

Ainsi le décrochage scolaire est devenu un enjeu majeur des politiques éducatives dans de nombreux pays. Dans un précédent numéro, un article appelait à dépasser le face-àface psychopédagogique (l'élève en échec) ou la critique des pratiques éducatives (l'exclu) pour inviter à une politique intersectorielle multiniveaux ${ }^{2}$. Ici, EL-Mahdi Khouaja et Stéphanie Moullet poursuivent l'analyse au travers d'une nouvelle typologie des élèves intégrant les caractéristiques des établissements dans le décrochage scolaire.

Tout comme le décrochage, la réussite scolaire, voire professionnelle, est loin d'être univoque ; dès lors, pour Sylvain Bourdon, María-Eugenia Longo et Johanne Charbonneau cohabitent des normes plus ou moins contradictoires. Identifier cette pluralité de normes permet d'isoler plusieurs figures de réussite, ici pour les jeunes de 17 à 19 ans en collège d'enseignement général et professionnel (Cégep) ${ }^{3}$ au Québec.

D'autant plus que les frontières du système éducatif sont loin d'être étanches. On pourrait attribuer certains facteurs de réussite à la formation, notamment en termes d'insertion, alors que de plus en plus d'étudiants sont en même temps salariés, et acquièrent ainsi des compétences valorisées ensuite. Ce phénomène semble prendre de plus en plus d'ampleur, comme dans l'exemple des diplômés universitaires catalans analysé par José NavarroCendejas et Jordi Planas Coll.

1 Marshall Rosenberg, Les mots sont des fenêtres (ou bien ce sont des murs), La Découverte, 2005.

2 Gérard Boudesseul, "De l'administration éducative du "décrochage scolaire" à la coopération intersectorielle. Vers un nouveau cadre de référence ?", in Qu'apprend-on des expérimentations sociales? Formation Emploi 126 | Avril-Juin 2014.

3 Les cégeps sont des établissements d'enseignement postsecondaire. Il s'agit de faire évoluer, dans un même établissement, des jeunes en formation technique (parcours de trois ans destinant au marché du travail) et en formation préuniversitaire (deux ans préparatoires à l'université) 
Une autre manière d'interroger les frontières de la formation est de s'intéresser à l'activité même des formateurs. A cet égard, comme le montre Iona Deicu, l'activité des formateurs en insertion s'adressant à des personnes en difficulté dépasse les seules préoccupations pédagogiques. Il devient important d'identifier la capacité d'adaptation des stagiaires à un environnement, d'entretenir des relations avec les prescripteurs, un réseau de partenaires, ou des employeurs potentiels.

On peut aussi interroger la frontière entre travail et hors travail (loisirs, famille) comme le font Christelle Marsault, Lilian Pichot et Julien Pierre. Pour les éducateurs sportifs, l'intrication des temps représente autant une contrainte qu'une ressource en fonction de leur identité professionnelle. La construction de cette dernière dépend de leurs ressorts personnels, mais aussi du contexte.

Pour leur part, Hervé Charmettant, Jean-Yves Juban, Nathalie Magne et Yvan Renou interrogent le contexte organisationnel dans le cadre des sociétés coopératives et participatives (Scop). Ici, la priorité est la sécurité de l'emploi. Cela conduit à des formes particulières de flexibilité et de participation des salariés, caractérisant une "sécuflexibilité » qui se veut durable et alternative à une flexicurité bien souvent présentée comme incontournable.

Bonne lecture. 